\title{
CDISC SEND Pre-Clinical Units of Measure Terminology
}

National Cancer Institute

\section{Source}

National Cancer Institute. CDISC SEND Pre-Clinical Units of Measure Terminology. NCI

Thesaurus. Code C77528.

The terminology that includes concepts relevant to CDISC SEND pre-clinical units of measure. 